\title{
Correlations between serum interleukin 6, serum soluble interleukin 6 receptor, and disease activity in systemic juvenile idiopathic arthritis patients treated with or without tocilizumab
}

\author{
SOAMARAT VILAIYUK ${ }^{1}$, BUTSABONG LERKVALEEKUL ${ }^{I}$, SIRISUCHA SOPONKANAPORN ${ }^{l}$, \\ CHAVACHOL SETTHAUDOM ${ }^{2}$, SUPRANEE BURANAPRADITKUN ${ }^{3}$
}

${ }^{1}$ Division of Rheumatology, Department of Pediatrics, Faculty of Medicine, Ramathibodi Hospital, Mahidol University, Bangkok, Thailand ${ }^{2}$ Immunology Laboratory, Department of Pathology, Faculty of Medicine, Ramathibodi Hospital, Mahidol University, Bangkok, Thailand ${ }^{3}$ Division of Allergy and Clinical Immunology, Department of Medicine, Faculty of Medicine, Chulalongkorn University, Bangkok, Thailand

\begin{abstract}
Introduction: Interleukin (IL)-6 is a proinflammatory cytokine involved in systemic juvenile idiopathic arthritis (SJIA). Since these patients are often treated with tocilizumab (TCZ), anti-IL-6 receptor (IL-6R) antibody, we investigated correlations between serum IL-6 and soluble IL-6R-levels and disease activity in SJIA patients treated with or without TCZ.

Material and methods: 164 serum samples were taken from 42 SJIA patients treated with or without TCZ (69 and 95 samples, respectively). Patients were assigned to three groups according to disease status: 1) systemic (patients with systemic features and/or arthritis), 2) arthritis (patients with arthritis but no systemic features), and 3) inactive (clinically inactive disease). Disease activity was assessed using the Juvenile Arthritis Disease Activity Score-27 (JADAS-27) at the time of blood collection.

Results: IL-6 levels were highest in SJIA patients with predominant systemic features, while serum sIL-6R levels were highest in patients with persistent arthritis. Serum IL-6 correlated with JADAS-27 in patients treated with and without TCZ ( $r=0.38$ and $r=0.65$, respectively), whereas serum sIL-6R levels correlated with JADAS-27 in patients treated without $(r=0.30)$ but not with $(r=-0.14)$ TCZ. The sIL-6R/IL-6 ratio negatively correlated with JADAS-27 in patients treated with and without TCZ ( $r=-0.49$ and $r=-0.56$, respectively).

Conclusions: Serum IL-6 levels correlated more strongly with disease activity parameters than did sIL-6R levels and could be useful for monitoring disease activity in SJIA patients. The sIL-6R/IL-6 ratio might be a promising disease activity marker in both SJIA patients treated with and without TCZ.
\end{abstract}

Key words: interleukin 6, interleukin 6 receptor, systemic juvenile idiopathic arthritis, juvenile idiopathic arthritis, tocilizumab, anti-interleukin 6 receptor antibody, correlation.

(Centr Eur J Immunol 2019; 44 (2): 150-158)

\section{Introduction}

Juvenile idiopathic arthritis (JIA), a common rheumatic disease in children, is classified into seven subtypes according to the International League of Associations for Rheumatology criteria [1]. Systemic JIA (SJIA) is a subtype with unique characteristics. Quotidian fever, salmon rash, hepatosplenomegaly, and serositis are also manifestations of SJIA, in addition to arthritis. Previous studies have classified SJIA as an autoinflammatory disease, and its pathogenesis has been reported to mainly involve innate rather than adaptive immunity [2], which contrasts with other JIA subtypes. Consistent with this, innate immunity cytokines, including interleukin (IL)-1 $\beta$, IL-6, IL-18, interferon $\gamma$, and tumour necrosis factor $\alpha$, play major roles in the pathogenesis of SJIA.

The proinflammatory cytokine IL- 6 contributes to many autoimmune diseases and is especially important in SJIA. IL-6 is a $21 \mathrm{kDa}$ glycoprotein that induces fever, leukocytosis, thrombocytosis, anaemia of chronic disease, and

Correspondence: Soamarat Vilaiyuk, MD, Division of Rheumatology, Department of Pediatrics, Faculty of Medicine, Ramathibodi Hospital, Mahidol University, 270 Rama VI Road, Ratchathewi, 10400 Bangkok, Thailand, e-mail: soamarat21@ hotmail.com Submitted: 10.01.2018; Accepted: 29.05.2018 
acute phase reactants, including C-reactive protein (CRP), erythrocyte sedimentation rate (ESR), and serum amyloid $\mathrm{A}$ [3]. IL-6 acts on numerous cell types, including B and $\mathrm{T}$ lymphocytes, hepatocytes, haematopoietic progenitor cells, macrophages, megakaryocytes, and neuronal cells [4-6]. The IL-6 receptor (IL-6R) complex consists of an $80 \mathrm{kDa}$ membrane-bound molecule (mIL-6R), a 50-55 kDa soluble molecule (sIL-6R) $[7,8]$, and a $130 \mathrm{kDa}$ membrane-bound glycoprotein (mgp130) that acts as the signal transducer [8-10]. Complex formation between IL- 6 and sIL-6R initiates the IL-6 trans-signalling pathway, whereas IL-6/mIL-6R complexes activate the classic IL-6R signalling pathway $[9,11]$. Binding of either complex to mgp130 induces homodimerisation and triggers a downstream signalling cascade $[12,13]$. A previous study by De Benedetti et al. revealed that serum IL-6 levels increased in SJIA patients during active disease and decreased with remission [14]. Furthermore, Yilmaz et al. found that serum IL-6 was elevated during both active and inactive disease, and they detected higher serum IL-6 levels in patients with SJIA compared with other JIA subtypes [15]. However, there are conflicting data regarding whether sIL-6R levels increase or decrease when SJIA is active $[16,17]$. Over the past decade, the humanised anti-human IL-6R monoclonal antibody tocilizumab (TCZ) has been developed for SJIA treatment, and it was included in the 2013 update of the 2011 American College of Rheumatology Recommendations for the Treatment of JIA [18]. Although some studies have investigated the relationship between TCZ treatment and serum IL-6 or sIL-6R levels, few have examined this in SJIA patients in the context of active and inactive disease. Therefore, in this study, we analysed the associations between serum IL-6 and sIL-6R levels and disease activity in SJIA patients treated with or without TCZ.

\section{Material and methods}

This study was approved by the Ethics Committee of Ramathibodi Hospital, Bangkok, Thailand, where the work was performed. The study was conducted in accordance with the ethical standards laid down in the Declaration of Helsinki (as revised in Brazil 2013), and written, informed consent was obtained from the patients' parents or legal guardians.

\section{Patients and samples}

This was a cross-sectional study. A total of 164 serum samples were obtained from 42 SJIA patients in Ramathibodi Hospital. Serum samples were obtained from each patient at three or four scheduled visits every two months between September 2012 and July 2013. Samples were assigned to one of three groups (see below) based on the patient's health status at the time of sample collec- tion. If the patient's disease status changed between visits, they were assigned to different groups. Samples were not collected if the patient had an infection at the time of the visit or during the preceding week. Fifteen control serum samples (one each from 15 healthy subjects) were included for comparison. Serum samples from SJIA patients treated with or without TCZ were assigned in TCZ and nonTCZ groups, respectively. TCZ was given to SJIA patients who were refractory to corticosteroid, or two conventional disease-modifying antirheumatic drugs. The dose of TCZ is $8 \mathrm{mg} / \mathrm{kg} / \mathrm{dose}$ intravenous infusion every two weeks. The serum samples from SJIA patients treated with TCZ were collected around two months after the initial treatment. Eighty-eight per cent of patients receiving TCZ had dramatic response to this medication, the inflammatory markers returned to normal, and systemic features subsided after the initial dose of TCZ. The rest of the patients, who were in the systemic group, started to respond to TCZ around 3-4 months after the initial dose.

\section{Data collection}

Baseline characteristics of SJIA patients included Juvenile Arthritis Disease Activity Score-27 (JADAS-27), active joint count, score for physician's global assessment (PGA), score for parent's or patient's global assessment of overall well-being (PtGA), and medications. Peripheral blood samples were sent for quantification of IL-6 and sIL-6R levels, complete blood counts, and ESR. Clinical assessments of disease status were performed at the time of sample collection. Samples were assigned to one of three groups based on the patient's clinical status: (1) systemic group: patients with active SJIA with systemic features (high-grade fever and at least one of the following: hepatosplenomegaly, serositis, salmon rash, and lymphadenopathy) with or without arthritis; (2) arthritis group: from patients with active SJIA with arthritis, but whose systemic features had subsided at least two months before each sample collection; and (3) inactive group: from patients whose systemic features and arthritis symptoms had subsided. Patients in the inactive group, some of whom were receiving medication, had normal ESR and CRP levels. JADAS-27 [19] was calculated as the sum of four variables assessed at the time of blood sample collection as follows: the total number of active joints ( 27 joints were assessed), the ESR normalised to a 0-10 scale according to ([ESR mm/h - 20] / 10), the PGA score (range 0-10), and the PtGA score (range 0-10). Thus, the minimum and maximum JADAS-27 were 0 and 57, respectively.

\section{IL-6 and sIL-6R measurements}

Serum was isolated from samples of 3-5 $\mathrm{ml}$ of blood and divided into two aliquots. One was stored at $-80^{\circ} \mathrm{C}$ until analysis of sIL-6R, while the second was used for measurement of IL-6 levels on the day of blood collection. 
IL-6 was quantified using an electrochemiluminescence immunoassay (Roche Diagnostics, Mannheim, Germany) that detects both free IL-6 and a complex of IL-6/sIL-6R. The lower limit of detection was $1.5 \mathrm{pg} / \mathrm{ml}$, and the normal level in this study was $\leq 7 \mathrm{pg} / \mathrm{ml}$. For measurement of serum IL-6R levels, the frozen sera were thawed to room temperature and analysed using an enzyme-linked immunosorbent assay (eBioscience, San Diego, CA, USA) that detects free sIL-6R, and complexes of IL-6/sIL-6R and TCZ/sIL-6R. The sensitivity of this test was $0.01 \mathrm{ng} / \mathrm{ml}$.

\section{Statistical analysis}

Baseline characteristics of SJIA disease activity and serum parameters were described as the median and interquartile range (IQR) or number and percentage of samples. A mixed model analysis was performed to assess differences among groups. Correlations between disease activity parameters and serum IL-6 or sIL-6R levels were analysed by Spearman's rank correlation test. Statistical significance was set at $p<0.05$. Data were analysed with Stata version 13 software (StataCorp, TX, USA).

\section{Results}

Of the 164 serum samples obtained, 69 and 95 were from patients treated with or without TCZ, respectively. Analysis of samples assigned to the three disease activity groups revealed that JADAS-27 scores were highest for patients in the systemic group (median [IQR] 18 [12.5]), followed by the arthritis group (5.9 [9.3]), and the inactive group (0 [0.8]) (Table 1). More patients in the systemic group than in the arthritis group received prednisolone, whereas the reverse was true for methotrexate treatment, consistent with standard treatment guidelines. The baseline characteristics of SJIA patients and serum parameters are shown in Table 1.

\section{Serum IL-6 levels in SJIA patients treated with or without $T C Z$}

Serum IL-6 levels were highest in the systemic group (median [IQR] 82.2 [102.3] pg/ml), followed by the arthritis group (19.9 [63.3] pg/ml), and the inactive group (6.7 [21.9] pg/ml). Significant differences were seen between IL-6 levels in the systemic group and the arthritis group $(p=0.004)$, inactive group $(p<0.001)$, and healthy control group (2.8 [1.9] pg/ml; $p=0.001$ ), as shown in Figure 1 .

TCZ treatment had no effect on the group order based on absolute serum IL- 6 levels. Thus, for patients treated with or without TCZ, the highest levels were seen in the systemic group, followed by the arthritis and inactive group (Table 2). However, within the arthritis subgroup, serum IL-6 levels were significantly higher in patients treated with than in those treated without TCZ (median [IQR] 57.3 [119.9] vs. 3.9 [22.4] pg/ml; $p<0.001$ ), whereas no TCZ-related differences were seen between patients within the systemic or inactive groups (Table 2).

\section{Serum soluble IL-6R levels in SJIA patients treated with or without $\mathrm{TCZ}$}

Overall, serum sIL-6R levels were highest in the arthritis group, followed by the inactive, systemic, and healthy control groups (Fig. 1). Levels in the arthritis group (median [IQR], 196.1 [971.6] ng/ml) and the inactive group (170.7 [1297.7] ng/ml), but not the systemic group (170.5 $[65.0] \mathrm{ng} / \mathrm{ml})$, were significantly higher than the levels in the healthy control group (66.5 [26.9] ng/ml), as shown in Figure 1.

Subgroup analysis showed that patients in the systemic and arthritic groups who did not receive TCZ had higher

Table 1. Baseline characteristics of systemic juvenile idiopathic arthritis patients at the time of serum sampling

\begin{tabular}{lccc}
\hline Characteristics & $\begin{array}{c}\text { Systemic } \\
\mathbf{n = 2 9 ( 1 6 )}\end{array}$ & $\begin{array}{c}\text { Arthritis } \\
\boldsymbol{n}=\mathbf{1 0 8}(\mathbf{3 6})\end{array}$ & $\begin{array}{c}\text { Inactive } \\
\boldsymbol{n}=\mathbf{2 7}(\mathbf{1 4})\end{array}$ \\
\hline Female (\% of patients) & 56.3 & 61.1 & 50.0 \\
\hline Age (years) & $6.0(4.75)$ & $7.0(6.0)$ & $6.0(8.3)$ \\
\hline Active joint & $6(11)$ & $2(4)$ & $0(0)$ \\
\hline PGA & $4.0(5.0)$ & $1.5(2.0)$ & $0(0)$ \\
\hline PtGA & $3.8(6.6)$ & $0.4(3.0)$ & $0(0)$ \\
\hline Haematocrit $(\%)$ & $33.2(5.3)$ & $36.2(3.7)$ & $37.4(3.8)$ \\
\hline Platelet $\times 10^{3}$ cells/ml & $483(227)$ & $380(152)$ & $343(131)$ \\
\hline ESR $(\mathrm{mm} / \mathrm{h})$ & $65(52)$ & $19(33)$ & $9(9)$ \\
\hline Prednisolone $(\%)$ & $22(76.0)$ & $55(51.0)$ & $3(11.1)$ \\
\hline Methotrexate $(\%)$ & $17(58.6)$ & $90(83.3)$ & $4(14.8)$
\end{tabular}

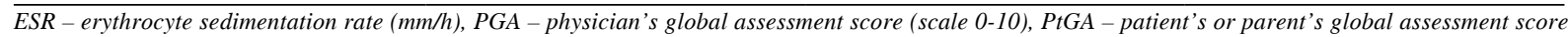
(scale 0-10), data are expressed as the median (interquartile range) or the number (\%) of samples 

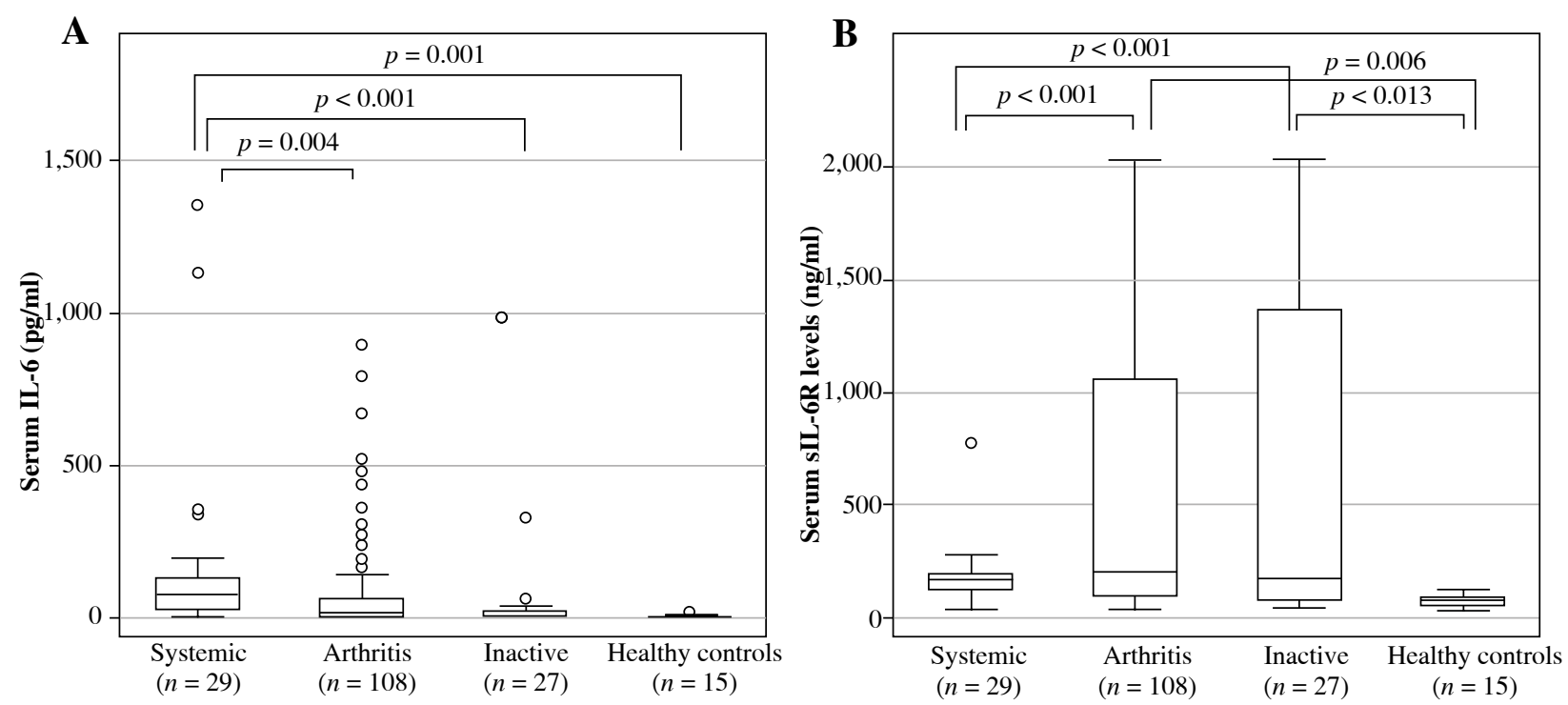

Fig. 1. Serum IL-6 and sIL-6R levels in systemic juvenile idiopathic arthritis patients. Patient serum samples were classified by disease status and analyzed for (A) serum IL-6 levels or (B) serum sIL-6R levels. Data are the median, interquartile range, and minimum/maximum values of the indicated number of samples for each subgroup

Table 2. Serum IL-6 and sIL-6R levels in systemic juvenile idiopathic arthritis patients treated with or without tocilizumab

\begin{tabular}{|c|c|c|c|c|c|c|c|c|}
\hline \multirow[t]{2}{*}{ Group } & \multicolumn{4}{|c|}{ Serum IL-6 levels (pg/ml) } & \multicolumn{4}{|c|}{ Serum sIL-6R levels (ng/ml) } \\
\hline & & $\begin{array}{l}\text { TCZ } \\
n\end{array}$ & & $\begin{array}{c}\text { TCZ } \\
n\end{array}$ & & $\begin{array}{l}\text { TCZ } \\
n\end{array}$ & & $\begin{array}{c}\text { TCZ } \\
n\end{array}$ \\
\hline Systemic & $23(14)$ & $85.1(165.6)$ & $6(3)$ & $79.0(66.1)$ & $23(14)$ & $149.7(72.5)$ & $6(3)$ & $218.7\left(83.6^{*}\right.$ \\
\hline Arthritis & $61(24)$ & $3.9(22.4)$ & 47 (17) & $57.3(119.9)^{*}$ & $61(24)$ & $95.6(91.7)$ & $47(17)$ & $1191.2(856.5)^{*}$ \\
\hline Inactive & $11(9)$ & $1.5(1.1)$ & $16(5)$ & $17.6(22.2)$ & $11(9)$ & $69.3(114.8)$ & $16(5)$ & $853.8(1566.6)^{*}$ \\
\hline
\end{tabular}

serum sIL-6R levels than healthy controls (149.7 [72.5], 95.6 [91.7], and 66.5 [26.9] ng/ml, respectively; $p<0.01)$. Moreover, within the TCZ-treated subgroup, serum sIL-6R levels were higher in samples from the arthritis and the inactive groups compared with the systemic group. In addition, sIL-6R levels were significantly higher for patients treated with than without TCZ for all three disease groups $(p<0.01)$ (Table 2). When the sIL-6R/IL-6 ratio was calculated in patients treated with and without TCZ (Fig. 2), patients in the systemic group had sIL-6R/IL-6 ratio lower than patients in the arthritis and the inactive groups in both SJIA patients treated with and without TCZ.

\section{Correlations between disease activity and serum IL-6 and sIL-6R levels}

Serum IL-6 levels were significantly correlated with JADAS-27 for patients treated both with and without TCZ (Table 3). In TCZ-untreated patients, serum IL-6 levels also correlated strongly (i.e. $r>0.6$ ) with PGA and ESR but moderately (i.e. $r=0.4-0.6$ ) with active joint counts and PtGA. In contrast, in TCZ-treated patients, serum IL-6 levels correlated weakly (i.e. $r<0.4$ ) with JADAS-27, active joint counts, PGA, and PtGA but showed no correlation with ESR. Serum sIL-6R levels correlated weakly with JADAS-27, active joint counts, PGA, and PtGA in TCZ-untreated patients. In TCZ-treated patients, sIL-6R levels had no correlation with disease activity parameters, except negative correlation with ESR (Table 3). The relationships between sIL-6R/IL-6 ratio and JADAS-27 and ESR were negatively correlated in both SJIA patients treated with and without TCZ as shown in Figure 3.

\section{Discussion}

This study focused on analysing serum IL-6 and sIL-6R levels in SJIA patients treated with or without TCZ. The results showed that both factors were higher in patients with active disease, including systemic features 


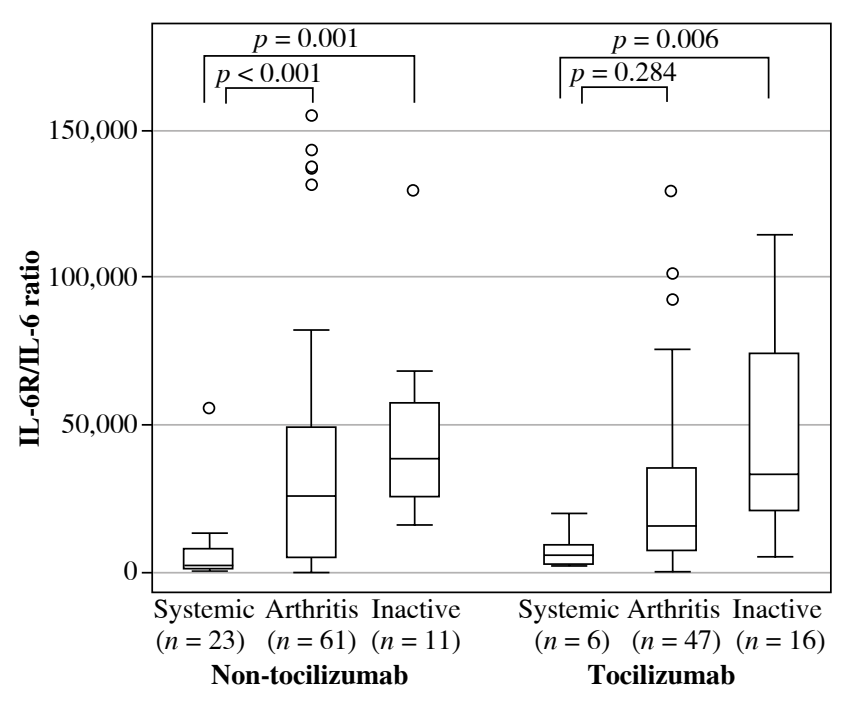

Fig. 2. sIL-6R/IL-6 ratio in systemic juvenile idiopathic arthritis (SJIA) patients treated with and without tocilizumab (TCZ). SJIA patient serum samples treated with and without TCZ were classified by disease status and analysed for sIL-6R/IL-6 ratio. Data are the median, interquartile range, and minimum/maximum values of the indicated number of samples for each subgroup

or/and arthritis, than in healthy controls. TCZ treatment also affected serum IL-6 and sIL-6R levels, with both being higher in patients treated with TCZ. Finally, serum IL-6 levels correlated more strongly than sIL-6R levels with disease activity parameters.

\section{IL-6 and TCZ treatment in SJIA patients}

Serum IL-6 levels are increased in many immuneinflammatory diseases and have been shown to correlate with disease activity [20-24]. Previous work showed that high levels of IL-6 in both serum and synovial fluid affected arthritis progression and correlated with clinical activity, including systemic features, growth retardation, and joint destruction [14, 25-29], suggesting that IL-6 affects systemic features, autoantibody production, and the expression of acute phase reactants $[30,31]$. In this study, we demonstrated that serum IL-6 levels reflected disease activity in SJIA patients, especially those with predominant systemic features. This could be explained by the widespread expression of mgp130 and high IL-6 and sIL-6R levels in patients with systemic features, resulting in activation of trans-signalling and classic IL-6 signalling pathways in multi-organ systems, not only in joints [4]. In 1991, De Benedetti et al. reported high serum IL-6 levels in patients with active disease, but the presence of systemic features did not affect the levels [14], which contrasts with our findings in this study. We showed that serum IL-6 levels were higher in SJIA patients with predominant systemic features than in those with persistent arthritis but no systemic features. Differences in patient populations may explain the discrepancy between the two studies. In the De Benedetti et al. study, patients with and without systemic features had similar disease status based on ESR and CRP values, whereas patients with predominant systemic features in this study had higher ESR and JADAS-27 - representative of more severe disease - than patients with persistent arthritis alone. We postulate that the regulation of IL-6 may differ in the two SJIA patient groups.

Serum IL-6 levels were only significantly affected by TCZ treatment in patients in the arthritis group. Within this group, $42.9 \%$ and $57.6 \%$ of serum samples were from patients treated with prednisolone with and without TCZ, respectively ( $p=0.09$ ), and $79.6 \%$ and $86.4 \%$ were from patients treated with methotrexate with or without TCZ, respectively $(p=0.44)$. The total number of patients taking these medications was similar in both arthritis subgroups. Therefore, it is more likely that treatment with TCZ, rather than methotrexate or prednisolone, is responsible for the difference in serum IL-6 levels between TCZ-treated and untreated patients within the arthritis group. IL-6 is eliminated via two pathways: IL-6R-mediated clearance, the main elimination pathway, and IL-6 degradation [32]. Binding of TCZ to IL-6R inhibits receptor-mediated clearance of IL-6, leading to its accumulation in serum. This is the likely explanation for the higher serum IL-6 levels in TCZ-treated SJIA patients in this study.

\section{Soluble IL-6R and TCZ treatment in SJIA patients}

We found that serum sIL-6R levels were significantly higher in the arthritis and inactive patient groups compared with the healthy control group. Previous studies have shown that IL-6R expression can be induced by IL-1 $\beta$ or IL-6, representing a positive feed-forward loop [33]. IL-6R is upregulated in human bronchial epithelial cells by IL-1 and IL-6 [34], and sIL-6R production is induced by IL-6 [35]. Therefore, the increase in serum sIL-6R levels observed in SJIA patients could be a direct consequence of increased serum IL-6.

In the TCZ-treated group, serum sIL-6R levels were lower in the systemic group compared to the arthritis and the inactive groups. When the sIL-6R/IL-6 ratio was calculated, SJIA patients in the systemic group had the lowest sIL-6R/IL-6 ratio compared to the other groups, whether receiving TCZ or not. The reason that sIL-6R levels and sIL-6R/IL-6 ratio were lower in the systemic group is currently unknown. A possible hypothesis could be explained 
Table 3. Correlations between disease activity parameters and serum IL-6, sIL-6R levels, and sIL-6R/IL-6 ratio

\begin{tabular}{lcccccc}
\hline Parameters & \multicolumn{5}{c}{ Spearman's coefficients } \\
\cline { 2 - 7 } & \multicolumn{2}{c}{ Serum IL-6 levels } & \multicolumn{2}{c}{ Serum sIL-6R levels } & \multicolumn{2}{c}{ Serum sIL-6R/IL-6 ratio } \\
\cline { 2 - 7 } & $\begin{array}{c}\text { No TCZ } \\
(\boldsymbol{n}=\mathbf{9 5})\end{array}$ & $\begin{array}{c}\text { TCZ } \\
(\boldsymbol{n}=\mathbf{6 9})\end{array}$ & $\begin{array}{c}\text { No TCZ } \\
(\boldsymbol{n}=\mathbf{9 5})\end{array}$ & $\begin{array}{c}\text { TCZ } \\
(\boldsymbol{n}=\mathbf{6 9})\end{array}$ & $\begin{array}{c}\text { No TCZ } \\
(\boldsymbol{n}=\mathbf{9 5})\end{array}$ & $\begin{array}{c}\text { TCZ } \\
(\boldsymbol{n}=\mathbf{6 9})\end{array}$ \\
\hline JADAS-27 & $0.65^{*}$ & $0.38^{*}$ & $0.30^{*}$ & -0.14 & $-0.56^{*}$ & $-0.49^{*}$ \\
\hline ESR & $0.66^{*}$ & -0.08 & 0.05 & $-0.64^{*}$ & $-0.64^{*}$ & $-0.34^{*}$ \\
\hline Active joint counts & $0.44^{*}$ & $0.33^{*}$ & $0.22^{*}$ & -0.06 & $-0.38^{*}$ & $-0.32^{*}$ \\
\hline PGA & $0.65^{*}$ & $0.38^{*}$ & $0.32^{*}$ & -0.05 & $-0.56^{*}$ & $-0.44^{*}$ \\
\hline PtGA & $0.48^{*}$ & $0.38^{*}$ & $0.30^{*}$ & 0.09 & $-0.37^{*}$ & $-0.36^{*}$ \\
\hline
\end{tabular}

IL-6 - interleukin 6, JADAS-27 - Juvenile Arthritis Disease Activity Score-27, PGA - physician's global assessment, PtGA - parent's or patient's global assessment, sIL-6R - soluble interleukin 6 receptor, sIL-6R/IL-6 ratio - sIL-6R divided by IL-6, ESR - erythrocyte sedimentation rate, ${ }^{*} p<0.05$
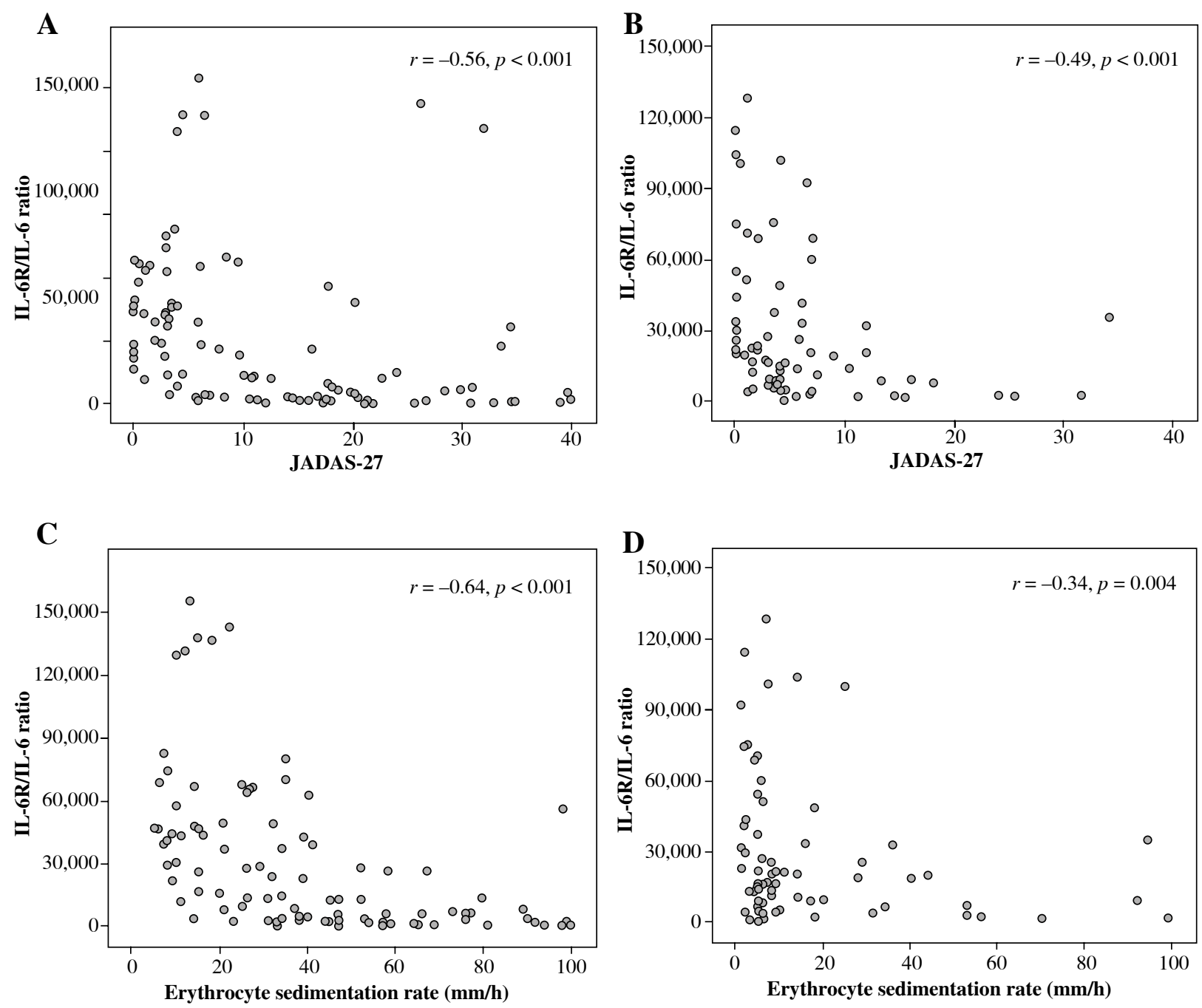

Fig. 3. Correlations between the sIL-6R/IL-6 ratio with Juvenile Arthritis Disease Activity Score-27 (JADAS-27) and erythrocyte sedimentation rate (ESR) in systemic juvenile idiopathic arthritis (SJIA) patients treated with and without tocilizumab (TCZ). Correlations between sIL-6R/IL-6 ratio with JADAS-27 in SJIA patients treated (A) without TCZ (95 samples) and (B) with TCZ (69 samples). Correlations between sIL-6R/IL-6 ratio with ESR in SJIA patients treated (C) without TCZ (95 samples) and (D) with TCZ (69 samples) 
by the fact that most sIL-6R in the systemic group might have already formed IL-6/sIL-6R/mgp130 complexes on the cell membranes, leading to inflammatory signals and systemic inflammation, as presented in patients in the systemic group. Because our sIL-6R ELISA could not detect IL-6/sIL-6R/mgp130 complexes, sIL-6R levels in the systemic group were lower than expected.

In another perspective, SJIA patients in the arthritis and the inactive groups did not have systemic features due to the blockage of sIL-6R by TCZ, resulting in the formation of TCZ/sIL-6R complexes. These TCZ/sIL-6R complexes caused a prolongation of the elimination half-life of sIL-6R. Consistent with this, sIL-6R levels began to increase upon commencement of TCZ treatment and remained high until discontinuation [32]. Because TCZ/sIL-6R complexes could be detected by our sIL-6R ELISA, this could explain why high levels of sIL-6R were found in the arthritis and the inactive groups. However, further studies with larger sample sizes will be necessary to confirm this finding and this hypothesis.

\section{Associations between SJIA disease activity and IL-6/sIL-6R}

In SJIA patients treated without TCZ, serum IL-6 levels showed good correlation with JADAS-27 and several parameters, including PGA and ESR, but only a moderate correlation with active joint counts. This was not unexpected, since the patients in this group had severe systemic features but only mild to moderate arthritis.

In SJIA patients treated with TCZ, serum IL-6 levels also correlated with JADAS-27 and several parameters, including PGA and active joint counts, but there was no correlation with ESR. This may be because binding of TCZ to mIL-6R or sIL-6R blocks IL-6 signalling and production of acute phase reactants by hepatocytes [36, 37]. In this scenario, ESR would be expected to decrease in TCZ-treated patients. In fact, acute phase reactants return to normal upon initiation of TCZ treatment, even though serum IL-6 levels remain high and arthritis remains active. This observation is supported by the correlations between IL-6 levels with active joint counts and PGA in patients treated with TCZ $(r=0.33, p=0.006$ and $r=0.38$, $p=0.001$, respectively) were higher than correlations between ESR with active joint counts and PGA in the same group of patients $(r=0.25, p=0.038$ and $r=0.20$, $p=0.102$, respectively). Thus, acute phase reactants may not be good markers for disease activity assessment, especially SJIA patients receiving TCZ with persistent arthritis but no systemic features. Serum IL-6 levels may be a useful marker of disease activity to complement clinical evaluation, especially in SJIA patients treated with TCZ.

In contrast, sIL-6R levels correlated only weakly with disease activity parameters, particularly in patients receiving TCZ. Nevertheless, this study demonstrated that sIL-
6R was strongly negatively correlated with ESR in SJIA patients receiving TCZ. Because TCZ had blocked IL-6 signalling and decreased production of acute phase reactants, ESR levels had returned to normal. At the same time, patients receiving TCZ had the complexes of TCZ/sIL-6R that prolonged elimination half-life of sIL-6R and caused high levels of sIL-6R in the blood stream. Therefore, these two factors were inversely correlated in patients receiving TCZ with clinically inactive disease. On the other hand, SJIA patients in the systemic group still had high levels of ESR and low levels of sIL-6R; this could also cause an inverse correlation. Although sIL-6R levels in SJIA patients treated with TCZ were negatively correlated with ESR, they did not correlate with JADAS-27. Therefore, using sIL-6R levels as a marker of disease activity might not be suitable. Furthermore, this study demonstrated negative correlations between sIL-6R/IL-6 ratio and disease activity parameters such as JADAS-27 and ESR, so the sIL-6R/ IL-6 ratio might be a useful marker of disease activity in the future. However, further studies are needed to confirm this finding.

The limitations of this study were 1) a small study population, especially in the systemic group treated with TCZ, and 2) a cross-sectional study design, which might not reflect changes in cytokine levels due to circadian rhythms in individual patients. Therefore, further studies with larger sample sizes and longitudinal study designs are recommended to verify these findings.

\section{Conclusions}

Serum IL-6 levels were highest in SJIA patients with predominant systemic features, while serum sIL-6R levels were highest in patients with persistent arthritis. TCZ treatment was associated with higher serum IL-6 and sIL$6 \mathrm{R}$ levels. There is an interesting finding that sIL-6R levels were lower in TCZ-treated patients with predominant systemic features than patients with only arthritis presentation and clinically inactive disease. Serum IL-6 levels correlated with disease activity parameters in all patients, with the exception of ESR in patients treated with TCZ. In addition, ESR correlated poorly with active joint count and was not correlated with PGA in TCZ-treated patients. Therefore, our results suggest that serum IL-6 levels may be a better marker than acute phase reactants for monitoring disease activity in SJIA patients, especially those treated with TCZ. In addition, we found no correlation between serum sIL-6R levels and disease activity parameters in SJIA patients receiving TCZ, except an inverse correlation with ESR, indicating that it should not be used to follow disease activity in these patients. Lastly, a promising marker, the sIL-6R/IL-6 ratio, might be useful for monitoring disease activity in both SJIA patients treated with and without TCZ. 


\section{Acknowledgements}

We thank the Faculty of Medicine at Ramathibodi Hospital for funding. We thank Dittapol Muntham and Nattawut Unwanatham (Section for Clinical Epidemiology and Biostatistics, Faculty of Medicine, Ramathibodi Hospital) and Dr. Suthat Chottanapund (Bamrasnaradura Infectious Diseases Institute) for statistical analysis. We greatly appreciate the valuable comments and suggestions of Prof. Pat Mahachoklertwattana (Department of Pediatrics, Faculty of Medicine, Ramathibodi Hospital) and Asst. Prof. Parawee Suwannalai and Prof. Sasisopin Kiertburanakul (Department of Medicine, Faculty of Medicine, Ramathibodi Hospital). We also thank Adam Dale, English language instructor at IGenius Language Institute, and Anne M. O'Rourke, PhD, from Edanz Group (www. edanzediting.com/ac) for editing drafts of this manuscript.

The authors declare no conflict of interest.

\section{References}

1. Petty RE, Southwood TR, Manners P, et al. (2004): International League of Associations for Rheumatology classification of juvenile idiopathic arthritis: second revision, Edmonton, 2001. J Rheumatol 31: 390-392.

2. Lin YT, Wang CT, Gershwin ME, Chiang BL (2011): The pathogenesis of oligoarticular/polyarticular vs systemic juvenile idiopathic arthritis. Autoimmun Rev 10: 482-489.

3. De Benedetti F (2009): Targeting interleukin-6 in pediatric rheumatic diseases. Curr Opin Rheumatol 21: 533-537.

4. Mihara M, Hashizume M, Yoshida H, et al. (2012): IL-6/IL-6 receptor system and its role in physiological and pathological conditions. Clin Sci 122: 143-159.

5. Scheller J, Rose-John S (2006): Interleukin-6 and its receptor: from bench to bedside. Med Microbiol Immunol 195: 173183.

6. Rose-John S, Scheller J, Elson G, Jones SA (2006): Interleukin- 6 biology is coordinated by membrane-bound and soluble receptors: role in inflammation and cancer. J Leukoc Biol 80: 227-236.

7. Hirano T, Yasukawa K, Harada H, et al. (1986): Complementary DNA for a novel human interleukin (BSF-2) that induces B lymphocytes to produce immunoglobulin. Nature 324: 73-76.

8. Yamasaki K, Taga T, Hirata Y, et al. (1988): Cloning and expression of the human interleukin-6 (BSF-2/IFN beta 2) receptor. Science 241: 825-828.

9. Hibi M, Murakami M, Saito M, et al. (1990): Molecular cloning and expression of an IL-6 signal transducer, gp130. Cell 63: 1149-1157.

10. Narazaki M, Yasukawa K, Saito T, et al. (1993): Soluble forms of the interleukin-6 signal-transducing receptor component gp130 in human serum possessing a potential to inhibit signals through membrane-anchored gp130. Blood 82: 1120-1126.

11. Jones SA, Richards PJ, Scheller J, Rose-John S (2005): IL-6 transsignaling: the in vivo consequences. J Interf Cytok Res 25: 241-253.
12. Murakami M, Hibi M, Nakagawa N, et al. (1993): IL-6induced homodimerization of gp130 and associated activation of a tyrosine kinase. Science 260: 1808-1810.

13. Heinrich P, Behrmann I, Haan S, et al. (2003): Principles of interleukin (IL)-6-type cytokine signalling and its regulation. Biochem J 374: 1-20

14. De Benedetti F, Massa M, Robbioni P, et al. (1991): Correlation of serum interleukin-6 levels with joint involvement and thrombocytosis in systemic juvenile rheumatoid arthritis. Arthritis Rheum 34: 1158-1163.

15. Yilmaz M, Kendirli SG, Altintas D, et al. (2001): Cytokine levels in serum of patients with juvenile rheumatoid arthritis. Clin Rheumatol 20: 30-35.

16. Keul R, Heinrich PC, Muller-Newen G, et al. (1998): A possible role for soluble IL-6 receptor in the pathogenesis of systemic onset juvenile chronic arthritis. Cytokine 10: 729-734.

17. De Benedetti F, Massa M, Pignatti P, et al. (1994): Serum soluble interleukin 6 (IL-6) receptor and IL-6/soluble IL-6 receptor complex in systemic juvenile rheumatoid arthritis. J Clin Invest 93: 2114-2119.

18. Ringold S, Weiss PF, Beukelman T, et al. (2013): 2013 update of the 2011 American College of Rheumatology recommendations for the treatment of juvenile idiopathic arthritis: recommendations for the medical therapy of children with systemic juvenile idiopathic arthritis and tuberculosis screening among children receiving biologic medications. Arthritis Rheum 65: 2499-2512.

19. Consolaro A, Ruperto N, Bazso A, et al. (2009): Development and validation of a composite disease activity score for juvenile idiopathic arthritis. Arthritis Rheum 61: 658-666.

20. Uson J, Balsa A, Pascual-Salcedo D, et al. (1997): Soluble interleukin 6 (IL-6) receptor and IL-6 levels in serum and synovial fluid of patients with different arthropathies. J Rheumatol 24: 2069-2075.

21. Yoshizaki K, Matsuda T, Nishimoto N, et al. (1989): Pathogenic significance of interleukin-6 (IL-6/BSF-2) in Castleman's disease. Blood 74: 1360-1367.

22. Mitsuyama K, Sasaki E, Toyonaga A, et al. (1991): Colonic mucosal interleukin-6 in inflammatory bowel disease. Digestion 50: 104-111.

23. De Benedetti F, Martini A (1998): Is systemic juvenile rheumatoid arthritis an interleukin 6 mediated disease? J Rheumatol 25: 203-207.

24. Yokota S (2003): Interleukin 6 as a therapeutic target in systemic-onset juvenile idiopathic arthritis. Curr Opin Rheumatol 15: 581-586.

25. Hermann E, Fleischer B, Mayet WJ, et al. (1989): Correlation of synovial fluid interleukin 6 (IL-6) activities with IgG concentrations in patients with inflammatory joint disease and osteoarthritis. Clin Exp Rheumatol 7: 411-414.

26. Houssiau FA, Devogelaer JP, Van Damme J, et al. (1988): Interleukin-6 in synovial fluid and serum of patients with rheumatoid arthritis and other inflammatory arthritides. Arthritis Rheum 31: 784-788.

27. Guerne PA, Zuraw BL, Vaughan JH, et al. (1989): Synovium as a source of interleukin 6 in vitro. Contribution to local and systemic manifestations of arthritis. J Clin Invest 83: 585-592.

28. De Benedetti F, Pignatti P, Gerloni V, et al. (1997): Differences in synovial fluid cytokine levels between juvenile and adult rheumatoid arthritis. J Rheumatol 24: 1403-1409.

29. Fonseca JE, Santos MJ, Canhao H, Choy E (2009): Interleukin-6 as a key player in systemic inflammation and joint destruction. Autoimmun Rev 8: 538-542. 
30. Desgeorges A, Gabay C, Silacci P, et al. (1997): Concentrations and origins of soluble interleukin 6 receptor-alpha in serum and synovial fluid. J Rheumatol 24: 1510-1516.

31. Madhok R, Crilly A, Watson J, Capell HA (1993): Serum interleukin 6 levels in rheumatoid arthritis: correlations with clinical and laboratory indices of disease activity. Ann Rheum Dis 52: 232-234.

32. Nishimoto N, Terao K, Mima T, et al. (2008): Mechanisms and pathologic significances in increase in serum interleukin-6 (IL-6) and soluble IL-6 receptor after administration of an anti-IL-6 receptor antibody, tocilizumab, in patients with rheumatoid arthritis and Castleman disease. Blood 112: 39593964.

33. Bauer J, Lengyel G, Bauer TM, et al. (1989): Regulation of interleukin-6 receptor expression in human monocytes and hepatocytes. FEBS Lett 249: 27-30.

34. Takizawa H, Ohtoshi T, Yamashita N, et al. (1996): Interleukin 6-receptor expression on human bronchial epithelial cells: regulation by IL-1 and IL-6. Am J Physiol 270: L346-L352.

35. Wolf J, Rose-John S, Garbers C (2014): Interleukin-6 and its receptors: a highly regulated and dynamic system. Cytokine 70: $11-20$.

36. Tanaka T, Narazaki M, Kishimoto T (2011): Anti-interleukin-6 receptor antibody, tocilizumab, for the treatment of autoimmune diseases. FEBS Lett 585: 3699-3709.

37. Gauldie J, Richards C, Harnish D, et al. (1987): Interferon beta $2 / \mathrm{B}$-cell stimulatory factor type 2 shares identity with monocyte-derived hepatocyte-stimulating factor and regulates the major acute phase protein response in liver cells. Proc Natl Acad Sci USA 84: 7251-7255. 